\title{
Psychosexual Aspects of Vulvovaginal Pain
}

Sophie Bergeron, Ph.D.

Associate Professor

Department of Psychology

Université de Montréal

Montréal, Québec, Canada

Wendy M. Likes, Ph.D.

Associate Professor

College of Nursing

College of Medicine, Department of Ob-gyn

University of Tennessee Health Science Center

Memphis, Tennessee, U.S.

Marc Steben, M.D.

Associate Professor

Department of Social and Preventive Medicine

Faculty of Medicine

Université de Montréal

Associate Physician

STI unit

Institut national de santé publique

Montréal, Québec, Canada

\section{Corresponding author:}

Sophie Bergeron, Ph.D.

Associate Professor

Department of Psychology

Université de Montréal

C.P. 6128, succursale Centre-Ville

Montréal, Québec

Canada, H3C 3J7

Tel: (514) 343-6111 \# 5353

Fax: (514) 343-2285

E-mail: sophie.bergeron.2@umontreal.ca 


\begin{abstract}
Vulvovaginal pain problems are major health concerns in women of childbearing age. Controlled studies have shown that vulvovaginal pain can adversely affect women and their partners' general psychological well-being, relationship adjustment and overall quality of life. These women have significantly lower levels of sexual desire, arousal, and satisfaction, as well as a lower intercourse frequency than normal controls. They also report more anxiety and depression, in addition to more distress about their body image and genital self-image. Empirical studies indicate that specific psychological and relationship factors may increase vulvovaginal pain intensity and its psychosexual sequelae. Randomized clinical trials have shown that psychosexual interventions, namely cognitive-behavioral therapy (CBT), are efficacious in reducing vulvovaginal pain and improving associated psychosexual outcomes. Women reporting significant psychological, sexual and/or relationship distress should be referred for psychosexual treatment. A multimodal approach to care integrating psychosexual and medical management is thought to be optimal.
\end{abstract}

Keywords: vulvovaginal pain, vulvodynia, provoked vestibulodynia, psychological adjustment, sexual function, cognitive-behavioral therapy 
Vulvovaginal pain problems are major health concerns in women of all ages. As conditions which are often misdiagnosed, mismanaged, trivialized or ignored, they entail a great personal cost to patients and a significant financial cost to society [1]. A case in point is vulvodynia: a population-based study suggests that the lifetime cumulative incidence of vulvodynia is $16 \%[2,3]$ and its incidence is thought to be increasing in young women $[4,5]$. In fact, many women suffering from vulvovaginal pain are under the age of 30, with community estimates showing that one in five women aged 18-29 report chronic dyspareunia [6]. In addition to disrupting all aspects of sexual function, controlled studies have shown that vulvovaginal pain can adversely affect women and their partners' general psychological well-being, relationship adjustment and overall quality of life [7, 8]. Because of their deleterious impact on sexuality and romantic relationships, vulvovaginal pain problems may indeed carry a heavier psychosocial burden in comparison to other pain problems common in women, with many patients reporting feelings of shame, inadequacy and low self-esteem [9]. Further, up to $45 \%$ of women with vulvodynia report a comorbid-pain condition, and having a comorbidity is associated with increased feelings of isolation and invalidation [10]. In terms of helpseeking behaviors, epidemiological results indicate that only $60 \%$ of women who report chronic vulvovaginal pain seek treatment, and $40 \%$ of these never receive a formal diagnosis [5]. A recent study involving a community sample showed that women saw an average of five physicians before diagnosis [10]. The quality of health care received by this patient population is thus less than optimal. It has been suggested that a multimodal approach taking into account not only the biomedical components of vulvovaginal pain 
but also its psychosexual aspects may constitute a promising avenue for managing this complex and multifaceted pain problem, as exemplified by the recent recommendations of the Third International Consultation on Sexual Medicine relating to women's sexual pain disorders [11].

\section{A. PSYCHOLOGICAL, SEXUAL AND RELATIONSHIP CONSEQUENCES}

Women with vulvovaginal pain show important impairments in many life domains, primarily their sexuality, intimate relationships and mental health. Specifically, controlled studies have shown that they report significantly less sexual desire, arousal and satisfaction, more difficulty reaching orgasm, as well as lower frequencies of intercourse and more negative attitudes toward sexuality than pain-free controls [12]. Research conducted in laboratory settings indicates that there are no significant differences between women with vulvovaginal pain and non afflicted women relative to their physiological level of sexual arousal when exposed to an erotic stimulus, although women with pain tend to report more negative feelings toward the stimulus [13-15].

Both quantitative and qualitative studies show that many women with vulvovaginal pain also report negative changes in their sexual self-esteem and in their body image, such as feeling less sexually desirable, feeling less confident about their sexuality and feeling less feminine than before [16-19]. Specifically, a study conducted among a community sample of 330 women showed that, in comparison to pain-free controls, women with vulvovaginal pain reported significantly more distress about their body image and a more negative genital self-image. Moreover, a more negative genital self-image was strongly and independently associated with an increased likelihood of 
reporting vulvovaginal pain, above and beyond anxiety [20]. In the same community sample of women with vulvovaginal pain, worse self-image cognitions about vaginal penetration contributed uniquely to increased pain intensity, whereas worse self-image cognitions about vaginal penetration and genital self-image contributed to poorer sexual functioning. Finally, worse self-image cognitions about vaginal penetration, body image and genital self-image each contributed independently to increased sexual distress [21]. These findings suggest that body image and genital self-image are significantly poorer in women with vulvovaginal pain and may influence key pain and sexuality outcomes.

Although there appear to be no differences in relationship satisfaction between women with vulvovaginal pain and asymptomatic controls, and no association between relationship satisfaction and pain in women with vulvovaginal complaints [22], this intimate pain nonetheless puts a significant strain on romantic relationships. Indeed, the fear of losing one's partner is noted across samples of women with vulvovaginal pain [23, 24]. It thus may come as no surprise that most afflicted women choose to continue engaging in vaginal penetration despite the pain and the limited satisfaction they derive from this sexual activity [25]. The question of why women continue to have sex despite vulvovaginal pain was asked to a group of sexually active young women and adolescents. Four reasons emerged: resignation, sacrifice, guilt, and the motivation to behave in a manner consistent with the notion of the ideal woman [26]. This situation may also become quite distressing for the partner, who may feel as though he is an incompetent lover and who may at times wonder whether he might be partly responsible for the pain. This may further complicate the dynamics of the relationship. In a study involving 38 vulvodynia couples, men's higher levels of both internal and global attributions for the 
pain - that is, thinking that the pain is their fault and that it affects all life domains were associated with their poorer relationship satisfaction, whereas global and stable attributions - thinking that the pain will never go away - were related to their lower sexual satisfaction [8].

As for mental health, several controlled studies involving clinical samples and using a wide variety of measures of depressive symptomatology have shown that women with vulvovaginal pain have higher depression scores than pain-free controls $[16,25,27$, 28], although three other controlled studies were unable to detect such a difference [12, $13,29]$. These contradictory findings could be attributed to the use of community samples in the latter studies, which typically report less distress. Many controlled studies have also found that these women display more state and trait anxiety than pain-free controls [30-32]. Further, there is evidence suggesting that anxiety may decrease the level of physiological sexual arousal in this population [15]. In summary, vulvovaginal pain results in several psychosexual sequelae, many of which require targeted interventions. Empirical work to date also suggests that specific psychosexual factors may maintain and exacerbate the different dimensions of vulvovaginal pain.

\section{B. PSYCHOSOCIAL PRECURSORS/MAINTENANCE FACTORS}

To date, controlled research has shown that compared to asymptomatic controls, women with vulvovaginal pain report more anxiety [28, 30,31, 33], depression [16] and pain catastrophizing. Higher levels of hypervigilance to pain, fear of pain, and catastrophizing have been found to be significant predictors of increased pain in this population, whereas greater anxiety and avoidance are associated with poorer sexual function. In addition, 
lower levels of pain self-efficacy, or the belief in one's capacity to meet the challenges of managing pain, are related to both worse pain and sexual function [34].

Three studies provide some evidence suggesting that psychosocial factors may contribute to the development of persistent vulvovaginal pain. A population-based survey of adult women found that sexual, physical and psychological abuse was linked to a 4 to 6-fold increased risk of reporting vulvodynia in adulthood [35, 36]. Another large-scale cross-sectional study focusing on adolescent girls with pain during intercourse showed that they reported significantly more sexual abuse and fear of physical abuse than controls [36]. Further, a community-based study showed that the odds of vulvodynia were 4 times more likely among women with antecedent depression or anxiety compared to women without [37]. However, because this area of research is still in its infancy, most work to date has been cross-sectional. Nonetheless, taken together, findings highlight the importance of environmental variables in the development of idiopathic vulvovaginal pain and indicate that psychosocial factors may contribute to the persistence of the pain and its associated negative sequelae.

Pain during intercourse is not limited to the woman's experience, but occurs in a relational context. In a first study on the dyadic aspects of provoked vestibulodynia conducted with 43 couples, greater partner solicitousness, i.e., demonstrations of sympathy, but also greater partner hostility, i.e. demonstrations of anger, were significantly associated with higher levels of pain during intercourse, suggesting that partner responses, both positive and negative, may play a role in the experience of vulvovaginal pain [38]. Results from a larger cross-sectional study of 191 couples indicated that higher solicitous partner responses - assessed from the perspective of both the 
woman and partner - were associated with higher levels of women's pain intensity.

Furthermore, the associations were mediated by greater patient and partner pain catastrophizing and self-efficacy $[39,40]$. In fact, partner pain catastrophizing and selfefficacy directly predicted patient pain, suggesting that the partner's view of the pain may modulate patient symptomatology [41]. Lower negative partner responses were also associated with more sexual satisfaction in women, and this association was mediated by greater dyadic adjustment $[39,40]$. Another study with a sample of 121 couples showed that facilitative partner responses - defined as partner responses that encourage a patient's efforts at adaptive coping with the pain (e.g., decreased avoidance) - were associated with lower vulvovaginal pain and higher sexual satisfaction [42]. Facilitative responses may be fostered in a targeted couple intervention by helping couples to focus on less painful activities, on the emotional benefits of sexual activity, and on expressing affection and pleasure during or after sexual activity.

Rosen et al. (in press) recently completed a two-month daily diary study involving 69 heterosexual couples coping with vulvodynia [43]. They found that a woman's pain during intercourse increased on days when she perceived greater solicitous and negative partner responses, and when her male partner reported greater solicitous and lower facilitative partner responses. A woman's sexual functioning and satisfaction increased on days when she perceived greater facilitative and lower solicitous and negative partner responses, and when her male partner reported lower solicitous responses. Further, a man's sexual functioning and satisfaction decreased on days when he reported greater solicitous and negative responses. These findings highlight the influence of partnerperceived variables on women's pain and sexual experience, as well as the impact of 
PVD on male partners' sexuality. To summarize, research findings have consistently shown that facilitative responses yield positive couple outcomes and should be fostered in an intervention, whereas solicitous and negative partner responses lead to negative outcomes and should be reduced.

Other recent research on relationship variables and vulvovaginal pain has focused on attachment, emotion regulation and intimacy. Attachment theory can provide a useful framework for understanding how couple dynamics may influence the experience of vulvovaginal pain and associated psychosexual difficulties. Attachment patterns are constructed within early interactions with primary caregivers [44]. Recent studies indicate that romantic attachment patterns are closely linked to sexual thoughts, emotions and behaviours [45, 46]. In a study involving 116 couples in which the women presented with provoked vestibulodynia, both anxious (anxiety about rejection) and avoidant (avoidance of intimacy) attachment styles were associated with women's lower sexual satisfaction. Attachment avoidance was associated with women's lower sexual function. Interestingly, women's sexual assertiveness was found to be a significant mediator of the relationship between their attachment styles, sexual function and satisfaction. This suggests that anxious and avoidant attachment may limit women's ability to be assertive in their sexuality, and that this lack of assertiveness may in turn lead to less satisfying sexual activities for these couples [47].

Indeed, how couples communicate about the vulvovaginal pain problem and its resulting negative consequences on their sex life could have an impact on their degree of adaptation to this challenge. A study conducted among 254 couples in which the woman suffered from vulvovaginal pain showed that those who had low ambivalence concerning 
the expression of their emotions, that is, couples who were the most comfortable with their level of expressiveness, had higher sexual satisfaction and sexual function, less depressive symptoms and better dyadic adjustment [47]. This suggests that an emotional regulation that is low in ambivalence for both members of the couple may promote better psychological, sexual and relational outcomes for women and their partners, possibly by facilitating their sharing about intimate aspects of the vulvovaginal pain problem and its impact on their sexuality.

Intimacy is another relationship variable which could help couples navigate the challenge of vulvovaginal pain. In a sample of 91 women diagnosed with provoked vestibulodynia and their partners, women's reported greater sexual intimacy was associated with women's increased sexual satisfaction and higher pain self-efficacy, beyond the effects of partners' sexual intimacy. Also, women's reported greater sexual intimacy and women's greater relationship intimacy were associated with their increased sexual function, beyond the effects of partners' sexual and relationship intimacy [48].

Taken together, findings concerning the psychosocial correlates of vulvovaginal pain suggest that the context within which this pain is experienced does matter. How women and their partners think, feel, communicate about this pain influences the extent to which they can adapt to it, reduce its intensity, and maintain a healthy sex life and relationship despite it.

\section{PSYCHOSEXUAL INTERVENTIONS}

The goals of most psychosexual interventions are to help patients view their vulvovaginal pain as a multidimensional problem influenced by a variety of factors including thoughts, 
emotions, behaviors and couple interactions; modify those factors associated with vulvovaginal pain with a view to increasing adaptive coping and decreasing pain intensity; improve the quality of their sexual functioning, including sexual desire which is often significantly low in this population, steering the focus away from intercourse while developing a more positive attitude toward other pleasurable sexual activities, and reduce avoidance of physical intimacy by working with their fear of pain. Mental health professionals working with these women also aim to facilitate adherence to other treatment regimens or procedures, such as gynaecological examinations or physical therapy.

Sex therapy and behavioral pain management combined together have been reported as successful in two studies focusing on dyspareunia, although these did not include control groups $[49,50]$. In the first randomized trial focusing on a psychological intervention for vulvovaginal pain, Bergeron et al. (2001) randomly assigned women with provoked vestibulodynia to a group cognitive-behavioral therapy (CBT) intervention, biofeedback or vestibulectomy. While findings indicated that vestibulectomy resulted in significant decreased pain outcomes among participants compared to the other two treatments, all three interventions showed equally enhanced sexual function and psychological adjustment outcomes. Treatment gains associated with CBT for PVD were maintained at 6-month and 2.5-year follow-ups for improvements in both pain and sexual functioning [51]. More importantly, women assigned to CBT did not differ from those assigned to vestibulectomy at the 2.5-year follow-up on the main outcome measure - pain during intercourse - prompting us to further investigate CBT. Post-treatment results from a second randomized trial show that women in the group CBT were significantly more 
satisfied with their treatment, displayed significantly less pain and pain catastrophizing, and reported significantly better global improvements in pain and sexual functioning than women assigned to a topical corticosteroid condition. These findings suggest that CBT may yield a positive impact on more dimensions of PVD than does some first-line medical treatments. Nevertheless, one limitation of these trials is that women's sexuality, although improved, remained within the clinical range of sexual dysfunction. This may be due to the fact that the partners were not involved. Another randomized trial examining the efficacy of individual CBT for vulvar pain compared to a supportive psychotherapy demonstrated that CBT yielded significantly greater improvements in pain and sexual function from pre- to post-treatment, with gains maintained at one-year follow-up [52]. Results of this study suggest a potential benefit for greater treatment outcome and patient satisfaction with a more directed approach such as CBT [52].

A systematic review of PVD treatment studies concluded that because behavioral treatments yield comparable success to several medical interventions but with no negative side effects, CBT represents an encouraging non-invasive option that can target pain as well as psychosexual consequences experienced by the woman and her partner [53]. However, CBT has been investigated successfully in a group and in an individual format, but never in a couple format for PVD, which is the most common and recommended way that CBT for sexual dysfunction is delivered in clinical settings.

\section{HOW TO IDENTIFY WOMEN WHO NEED PSYCHOSEXUAL}

\section{COUNSELING}


Women who report significant sexual impairment, sometimes to the point of abstaining from all partnered sexual activities, may need more than a medical intervention to surmount the vicious cycle of pain and avoidance that they are caught in. Significant depressive and anxiety symptoms, that would be verbalized as discouragement, not seeing the light at the end of the tunnel and feeling like a hopeless case, also warrant a psychological assessment and possibly psychotherapy combined with medical treatment. Anger at health care professionals, although expected and understandable to some degree, may constitute another red flag when displayed at more intense and sustained levels despite the repeatedly empathic stance of the caregiver. Significant relationship difficulties also need to be addressed in couple therapy, especially since they may interfere with ongoing treatment, for example in the case of a partner who sabotages the woman's efforts at improving her condition, or simply does not support her in any way. In addition, women with pre-existing relationship or sexual difficulties could be in need of couple therapy to cope with the added burden of pain. Finally, women who have tried multiple treatments and have not benefited from any single intervention, or women who have experienced many negative side effects from a medical treatment or complications from surgery, may have underlying psychological predispositions that prevent them from deriving all the positive effects typically associated with a given modality. Indeed, higher levels of pre-treatment pain catastrophizing and lower pre-treatment levels of pain selfefficacy have been shown to predict, at 6-months post-treatment, worse outcomes following both cognitive-behavioral therapy and a topical application [54]. Certain personality traits or attachment difficulties could also make it more difficult for some patients to develop a trusting relationship with their health care provider, which is 
thought to be a key ingredient of any successful treatment, particularly in the context of a multimodal approach [55].

\section{E. A MULTIDISCIPLINARY APPROACH TO CARE}

There are only two published studies evaluating a multimodal approach to the treatment of vulvovaginal pain, both of which involved psychosexual counselling and pelvic floor rehabilitation in addition to the usual medical care provided by a physician or nurse. In their uncontrolled, prospective investigation, Backman, Widenbrant, Bohm-Starke, \& Dahlof [56] found that $67 \%$ of 24 participants reported occasional or mild pain at sixmonth follow-up, while $63 \%$ reported a major improvement in their sexual function. Using a retrospective questionnaire survey 3-7 years post-treatment, Spoelstra, Dijkstra, van Driel \& Weijmar Schultz [57] showed that $80 \%$ of women had resumed intercourse and $81 \%$ reported pain reduction, although only $8 \%$ reported completely pain-free intercourse. These findings are encouraging and suggest the need for more empirical work aimed at assessing the efficacy of an integrated approach to care.

From a clinical standpoint, advantages of this model are numerous and often include an accelerated, more focused treatment process, less patient resistance to any one single biomedical or psychosocial intervention, more motivated and persistent patients and health care providers, increased coherence among the various health disciplines involved, and multiple facets of vulvovaginal pain being targeted simultaneously [58].

Nonetheless, despite its high prevalence, health professionals trained in the treatment of vulvovaginal pain are a rarity in some large cities of developed countries, let alone in rural areas and in other parts of the world, making patient access to a multimodal 
approach to care often difficult, if not altogether impossible. Telemedicine and einterventions show promise in reaching remote populations of women. Self-help groups may also provide patients with much needed support and serve to disseminate knowledge amongst themselves. In sum, there is a strong need for more integrated health care services addressing the psychosexual dimensions of vulvovaginal pain, in order for a larger proportion of afflicted women and couples to break out of their isolation and to receive the care and pain relief they deserve.

\section{SUMMARY}

Vulvovaginal pain has a significant negative impact on the sexual function and psychological adjustment of afflicted women and their partners. Cross-sectional and daily diary studies show that specific psychological factors can modulate pain and psychosexual sequelae. Individual variables include higher pain catastrophizing, hypervigilance to pain, fear of pain, anxiety and avoidance, and lower pain self-efficacy. Interpersonal factors include spouse solicitous and negative responses, poorer sexual and relationship intimacy, anxious and avoidant attachment styles, and higher ambivalence over emotional expression. There is growing evidence that cognitive-behavioral therapy a treatment targeting these different psychological factors - can effectively reduce vulvovaginal pain and associated psychosexual difficulties. Combining this type of care with concurrent medical management is thought to represent the most optimal model for treating vulvovaginal pain and the significant distress that it generates in patients and their partners, although this model has not been validated empirically. 


\section{Practice points}

- Women with vulvovaginal pain show significantly more psychological distress and sexual dysfunction than normal controls

- Women with antecedent anxiety, depression and/or sexual abuse may be more at risk of developing vulvovaginal pain

- Psychological and relationship factors modulate pain and associated psychosexual difficulties

- Cognitive-behavioral therapy is an effective treatment for vulvovaginal pain

- Including the partner in any type of treatment for vulvovaginal pain can make a difference in key patient outcomes

\section{Research agenda}

- Randomized clinical trials to test the efficacy of couple cognitive-behavioral therapy and of a multimodal approach to care

- Predictors and mediators of psychosexual treatment outcomes

- Daily diary and prospective designs to identify temporal patterns of relations between psychosocial factors, pain and psychosexual difficulties

- Longitudinal research in community samples of pre-pubescent girls followed through adulthood to test hypothesized causal relations between childhood trauma, hypothalamo-pituitary-adrenal axis dysregulation, and vulvovaginal pain

\section{Multiple choice questions}




\section{Question 1}

Why do women continue to engage in sex when vulvovaginal pain is present?

(a) guilt

(b) sacrifice

(c) fear

(d) resignation

\section{$\underline{\text { Answers to question } 1}$}
(a) $\mathrm{T}$
(b) $\mathrm{T}$
(c) $\mathrm{F}$
(d) $\mathrm{T}$

\section{Explanation to the answers to question 1}

A study by Elmerstig et al. (2008) [26] found women report guilt, sacrifice, resignation, and the motivation to behave in a manner consistent with the notion of an ideal woman as reasons for why women continue to have sex despite the presence of pain.

\section{Question 2}

In terms of help-seeking behaviors, what percentage of women who report chronic vulvovaginal pain receive a formal diagnosis?
(a) $85 \%$
(b) $60 \%$
(c) $36 \%$
(d) $40 \%$

\section{Answers to question 2}



(a) $\mathrm{F}$
(b) F (c) $\mathrm{T}$
(d) F

\section{Explanation to the answers to question 2}

Epidemiological results indicate that only $60 \%$ of women who report

chronic vulvovaginal pain seek treatment, and $40 \%$ of these never receive a formal diagnosis [ㄷ].

\section{Question 3}

Are there significant differences between women with vulvovaginal pain and non afflicted women relative to their physiological level of sexual arousal when exposed to an erotic stimulus?

(a) Yes, women with pain are less aroused.

(b) Yes, women with pain report more negative feelings toward the stimulus.

(c) No, women with pain do not report more negative feelings toward the stimulus.

(d) No, women with pain are equally aroused.

\section{Answers to question 3}
(a) $\mathrm{F}$
(b) $\mathrm{T}$
(c) $\mathrm{F}$
(d) $\mathrm{T}$

\section{Explanation to the answers to question 3}

Research conducted in laboratory settings indicates that there are no significant differences between women with vulvovaginal pain and non afflicted women relative to their physiological level of sexual arousal when exposed to an erotic stimulus, although women with pain tend to report more negative feelings toward the stimulus [13-15]. 


\section{REFERENCES}

1. Walling MK \& Reiter RC. Chronic pelvic pain. In O'Hara, MW et al. (eds) Psychological Aspects of Women's Reproductive Health. pp 65-80. New York: Springer Publishing Company, 1995.

2. Harlow BL \& Stewart EG. A population-based assessment of chronic unexplained vulvar pain: Have we underestimated the prevalence of vulvodynia? $\underline{\mathrm{J} \text { Am Med }}$ Womens Assoc 2003; 58: 82-88.

3. Canada, S. Estimations de la population selon le sexe et le groupe d'âge au ler juillet 2011, Canada. 2011; Available from: http://www.statcan.gc.ca/dailyquotidien/110928/t110928a4-fra.htm.

4. Danielsson I, Sjöberg I, Stenlund H \& Wikman M. Prevalence and incidence of prolonged and severe dyspareunia in women: Results from a population study. Scand J Public Health 2003; 31: 13-18.

5. Harlow BL, Wise LA \& Stewart EG. Prevalence and predictors of chronic lower genital tract discomfort. Am J Obstet Gynecol 2001; 185: 545-550.

6. Laumann EO, Paik A \& Rosen RC. Sexual dysfunction in the United States: Pevalence and predictors. JAMA 1999; 281: 537-544.

7. Arnold LD, Bachmann GA, Rosen R et al. Vulvodynia: Characteristics and associations with comorbidities and quality of life. Obstet Gynecol 2006; 107: 617-624. 
8. Jodoin M, Bergeron S, Khalifé S et al. Male partners of women with provoked vestibulodynia: Attributions for pain and their implications for dyadic adjustment, sexual satisfaction, and psychological distress. J Sex Med 2008; 5: 2862-2870.

9. Desrochers G, Bergeron S, Landry T \& Jodoin M. Do psychosexual factors play a role in the etiology of provoked vestibulodynia? A critical review. J Sex Marital Ther 2008; 34: 98-226.

10. Nguyen RH, Ecklund AM, Maclehose RF et al. Co-morbid pain conditions and feelings of invalidation and isolation among women with vulvodynia. $\underline{\text { Psychol }}$ Health Med 2012; 17: 589-598.

11. van Lankveld JJ, Granot M, Weijmar Schultz WC et al. Women's sexual pain disorders. J Sex Med 2010; 7: 615-631.

12. Meana M, Binik YM, Khalife S \& Cohen DR. Biopsychosocial profile of women with dyspareunia. Obstet Gynecol 1997; 90: 583-589.

13. Payne KA, Binik YM, Pukall CF et al. Effects of sexual arousal on genital and non-genital sensation: A comparison of women with vulvar vestibulitis syndrome and healthy controls. Arch Sex Behav 2007; 36: 289-300.

14. Brauer M, Laan E \& ter Kuile MM. Sexual arousal in women with superficial dyspareunia. Arch Sex Behav 2006; 35: 191-200.

15. Brauer M, ter Kuile MM, Janssen E \& Laan E. The effect of pain-related fear on sexual arousal in women with superficial dyspareunia. Eur J Pain 2007; 11: 788798.

16. Gates EA \& Galask RP. Psychological and sexual functioning in women with vulvar vestibulitis. J Psychosom Obstet Gynaecol 2001; 22: 221-228. 
17. Sackett S, Gates E, Heckman-Stone C et al. Psychosexual aspects of vulvar vestibulitis. J Reprod Med 2001; 46: 593-8.

18. Reed BD, Advincula AP, Fonde KR et al. Sexual activities and attitudes of women with vulvar dysesthesia. Obstet Gynecol 2003; 102: 325-31.

19. Ayling K \& Ussher JM. "If sex hurts, am I still a woman?" the subjective experience of vulvodynia in hetero-sexual women. Arch Sex Behav 2008; 37: 294-304.

20. Pazmany E, Bergeron S, Van Oudenhove L et al. Body image and genital selfimage in pre-menopausal women with dyspareunia. Arch Sex Behav 2013; 42: 999-1010.

21. Pazmany E, Bergeron S, Van Oudenhove L et al. Aspects of sexual self-schema in premenopausal women with dyspareunia: Associations with pain, sexual function, and sexual distress. J Sex Med 2013.

22. Smith KB \& Pukall CF. A systematic review of relationship adjustment and sexual satisfaction among women with provoked vestibulodynia. J Sex Res 2011; 48: 166-191.

23. Gordon AS, Panahian-Jand M, Mccomb F et al. Characteristics of women with vulvar pain disorders: Responses to a Web-based survey. J Sex Marital Ther 2003; 29: 45-58.

24. Sheppard C, Hallam-Jones R \& Wylie K. Why have you both come? Emotional, relationship, sexual and social issues raised by heterosexual couples seeking sexual therapy (in women referred to a sexual difficulties clinic with a history of vulval pain). Sex Relation Ther 2008; 23: 217-226. 
25. Hallam-Jones R, Wylie KR, Osborne-Cribb J et al. Sexual difficulties within a group of patients with vulvodynia. Sex Relation Ther 2001; 16: 113-126.

26. Elmerstig E, Wijma B \& Berterö C. Why do young women continue to have sexual intercourse despite pain. J Adolesc Health 2008; 43: 357-363.

27. Reed BD, Haefner HK, Punch MR, et al. Psychosocial and sexual functioning in women with vulvodynia and chronic pelvic pain. A comparative evaluation. $\underline{\mathbf{J}}$ Reprod Med 2000; 45: 624-32.

28. Lundqvist EN \& Bergdahl J. Vulvar vestibulitis: Evidence of depression and state anxiety in patients and partners. Acta Derm Venereol 2003; 83: 369-373.

29. Aikens JE, Reed BD, Gorenflo DW \& Haefner HK. Depressive symptoms among women with vulvar dysesthesia. Am J Obstet Gynecol 2003; 189: 462-466.

30. Granot M \& Lavee Y. Psychological factors associated with perception of experimental pain in vulvar vestibulitis syndrome. J Sex Marital Ther 2005; 31: 285-302.

31. Payne KA, Binik YM, Amsel R \& Khalifé S. When sex hurts, anxiety and fear orient attention towards pain. Eur J Pain 2005; 9: 427-436.

32. Landry T \& Bergeron S. Biopsychosocial factors associated with dyspareunia in a community sample of adolescent girls. Arch Sex Behav 2011; 40: 877-889.

33. Granot M, Friedman M, Yarnitsky D \& Zimmer EZ. Enhancement of the perception of systemic pain in women with vulvar vestibulitis. BJOG 2002; 109: 863-866. 
34. Desrochers G, Bergeron S, Khalifé S et al. Fear avoidance and self-efficacy in relation to pain and sexual impairment in women with provoked vestibulodynia. Clin J Pain 2009; 25: 520-527.

35. Harlow BL \& Stewart EG. Adult-onset vulvodynia in relation to childhood violence victimization. Am J Epidemiol 2005; 161: 871-880.

36. Landry T \& Bergeron S. Biopsychosocial factors associated with dyspareunia in a community sample of adolescent girls. Arch Sex Behav 2011; 40: 877-89.

37. Khandker M, Brady SS, Vitonis AF et al. The influence of depression and anxiety on risk of adult onset vulvodynia. J Womens Health 2011; 20: 1445-1451.

38. Desrosiers M, Bergeron S, Meana M et al. Psychosexual characteristics of vestibulodynia couples: Partner solicitousness and hostility are associated with pain. J Sex Med 2008; 5: 418-427.

39. Rosen NO, Bergeron S, Leclerc B et al. Woman and partner-perceived partner responses predict pain and sexual satisfaction in provoked vestibulodynia (PVD) couples. J Sex Med 2010; 7: 3715-3724.

40. Rosen NO, Bergeron S, Lambert B \& Steben M. Provoked vestibulodynia: Mediators of the associations between partner responses, pain, and sexual satisfaction. Arch Sex Behav 2013; 42: 129-141.

41. Lemieux AJ, Bergeron S, Steben M \& Lambert B. Do romantic partners' responses to entry dyspareunia affect women's experience of pain? The roles of

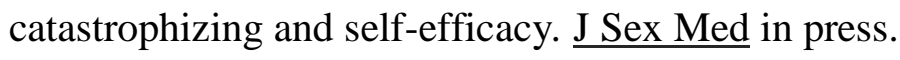


42. Rosen NO, Bergeron S, Glowacka M et al. Harmful or helpful: Partner responses are differentially associated with pain, sexual satisfaction and dyadic adjustment in women with provoked vestibulodynia. J Sex Med 2012; 9: 2351-2360.

43. Rosen NO, Bergeron S, Sadikaj G et al. Partner responses and sexual function in women with vulvodynia and their partners: A dyadic daily experience study. Health Psychol in press.

44. Mikulincer M \& Goodman GS. In Dynamics of romantic love: Attachment, caregiving, and sex. Guilford Press: New York, 2006.

45. Cooper ML, Pioli M, Levitt A et al. Attachment styles, sex motives and sexual behavior: Evidence for gender specific expressions of attachment dynamics. In Mikulincer M \& Goodman GS (eds) Dynamics of Love: Attachment, Caregiving, and Sex. pp 243-273. Guilford Press: New York, 2006.

46. Dewitte M. Different perspectives on the sex-attachment link: Towards an emotion-motivational account. J Sex Res 2012; 49: 105-24.

47. Awada N, Corsini-Munt S, Rosen N \& Bergeron S. Sexual anxiety. In P.E.T.E. (eds) International Handbook of Anxiety Disorders: Theory, Research and Practice I. Wiley-Blackwell: Chichester, in press.

48. Bois K, Bergeron S, Rosen NO, et al. Sexual and relationship intimacy among women with provoked vestibulodynia and their partners: Associations with sexual satisfaction, sexual function, and pain self-efficacy. J Sex Med 2013; 10: 20242035.

49. Weijmar Schultz WC, Gianotten WL, van der Meijden WI et al. Behavioral approach with or without surgical intervention to the vulvar vestibulitis syndrome: 
A prospective randomized and non-randomized study. $\underline{\mathrm{J} \text { Psychosom Obstet }}$

Gynaecol 1996; 17: 143-148.

50. ter Kuile MM \& Weijenborg PT. A cognitive-behavioral group program for women with vulvar vestibulitis syndrome (VVS): Factors associated with treatment success. J Sex Marital Ther 2006; 32: 199-213.

51. Bergeron S, Khalifé S, Glazer HI \& Binik YM. Surgical and behavioral treatments for vestibulodynia: Two-and-one-half year follow-up and predictors of outcome. Obstet Gynecol 2008; 111: 159-166.

52. Masheb RM, Kerns RD, Lozano C et al. A randomized clinical trial for women with vulvodynia: Cognitive-behavioral therapy vs. supportive therapy. Pain 2009; 141: $31-40$.

53. Landry T, Bergeron S, Dupuis MJ \& Desrochers G. The treatment of provoked vestibulodynia: A critical review. Clin J Pain 2008; 24: 155-171.

54. Desrochers G, Bergeron S, Khalifé S et al. Provoked vestibulodynia: Psychological predictors of topical and cognitive-behavioral treatment outcome. Behav Res Ther 2010; 48: 106-115.

55. Bergeron S, Landry T \& Leclerc B. Psychological and alternative treatments. In Goldstein A, Pukall CF \& Goldstein I. (eds) Female Sexual Pain Disorders: Evaluation and Management. pp150-155. Blackwell Publishing: Oxford, 2009.

56. Backman H, Widenbrant M, Bohm-Starke N \& Dahlof LG. Combined physical and psychosexual therapy for provoked vestibulodynia-an evaluation of a multidisciplinary treatment model. J Sex Res 2008; 45: 378-385. 
57. Spoelstra SK, Dijkstra JR, van Driel MF \& Weijmar Schultz WC. Long-term results of an individualized, multifaceted, and multidisciplinary therapeutic approach to provoked vestibulodynia. J Sex Med 2011; 8: 489-496.

58. Sadownik LA, Seal BN \& Brotto LA. Provoked vestibulodynia : Women's experience of participating in a multidisciplinary vulvodynia program. $\underline{\mathrm{J} \text { Sex Med }}$ 2012; 9: 1086-1093. 